\title{
Bioanalysis
}

\section{White Paper on recent issues in bioanalysis: focus on biomarker assay validation (BAV): (Part 2 - Hybrid LBA/ LCMS and input from regulatory agencies)}

The 2016 10th Workshop on Recent Issues in Bioanalysis (10 ${ }^{\text {th }}$ WRIB) took place in Orlando, Florida with participation of close to 700 professionals from pharmaceutical/ biopharmaceutical companies, biotechnology companies, contract research organizations, and regulatory agencies worldwide. WRIB was once again a 5-day, weeklong event - A Full Immersion Week of Bioanalysis including Biomarkers and Immunogenicity. As usual, it is specifically designed to facilitate sharing, reviewing, discussing and agreeing on approaches to address the most current issues of interest including both small and large molecules involving LCMS, hybrid LBA/LCMS, and LBA approaches, with the focus on biomarkers and immunogenicity. This 2016 White Paper encompasses recommendations emerging from the extensive discussions held during the workshop, and is aimed to provide the bioanalytical community with key information and practical solutions on topics and issues addressed, in an effort to enable advances in scientific excellence, improved quality and better regulatory compliance. This White Paper is published in 3 parts due to length. This part (Part 2) discusses the recommendations for Hybrid LBA/LCMS and regulatory inputs from major global health authorities. Parts 1 (small molecule bioanalysis using LCMS) and Part 3 (large molecule bioanalysis using LBA, biomarkers and immunogenicity) have been published in the Bioanalysis journal, issues 22 and 23, respectively.

First draft submitted: 30 September 2016; Accepted for publication: 3 October 2016; Published online: 18 November 2016

\section{Introduction}

This year marked the 10 th anniversary edition of the Workshop on Recent Issues in Bioanalysis (10th WRIB), which was held in Orlando, Florida from April 18-22, 2016. Nearly 700 professionals from pharmaceutical/biopharmaceutical companies, biotechnology companies, contract research organizations (CROs), and regulatory agencies worldwide were in attendance. In addition to the three focused and sequential workshop days, there were six advanced training sessions throughout the week providing attendees multiple choices to combine core workshop days and training, thereby maximizing their learning process in many fields of drug development expertise. As with prior WRIBs, this 10th edition was specifically designed to facilitate sharing, reviewing, discussing and agreeing upon scientific and regulatory approaches to address the most recent issues in both small and large molecule bioanalysis, biomarkers and immunogenicity. The areas of interest included LCMS, hybrid LBA/ LCMS as well as LBA/cell-based approaches.

The chairs of the 2016 edition of the WRIB included Dr Eric Yang (GlaxoSmithKline), Dr Jan Welink (EMA), Dr An Song (Genentech), Dr Fabio Garofolo (Angelini Pharma), Dr Susan Richards (Sanofi), Dr Lakshmi Amaravadi (Sanofi) and Dr Renuka Pillutla (Bristol-Myers Squibb).

As usual, a number of regulatory agency representatives contributed actively to the 10th WRIB, including Dr Sam Haidar (US FDA), Dr Nilufer Tampal (US FDA), Dr John Kadavil (US FDA), Dr Kara Scheibner (US FDA), Dr João Pedras-Vasconcelos (US FDA), Dr Jan Welink (EU EMA), Dr Ronald Bauer (Austria AGES), Mr Jason Wake-
An Song ${ }^{1}$, Anita Lee ${ }^{2}$, Fabio Garofolo*3, Surinder Kaur ${ }^{1}$, Jeff Duggan ${ }^{4}$, Christopher Evans $^{5}$, Joe Palandra ${ }^{6}$, Lorella Di Donato7, Keyang $\mathrm{Xu}^{1}$, Ronald Bauer, Mark Bustard $^{9}$, Linzhi Chen ${ }^{4}$, Laurent Cocea ${ }^{9}$, Stephanie Croft $^{10}$, Fabrizio Galliccia ${ }^{11}$, Sam Haidar ${ }^{12}$, Nicola Hughes $^{13}$, Akiko IshiiWatabe $^{14}$, Rafiqul Islam ${ }^{15}$, Barry Jones $^{16}$, John Kadavil ${ }^{12}$, Carsten Krantz' ${ }^{17}$, Gustavo Mendes Lima Santos ${ }^{18}$, Timothy Olah ${ }^{19}$, João PedrasVasconcelos ${ }^{12}$, Ludovicus Staelens ${ }^{20}$, Yoshiro Saito ${ }^{14}$, Natasha Savoie ${ }^{21}$, Kara Scheibner ${ }^{12}$, Susan Spitz ${ }^{22}$, Nilufer Tampal'2, Eric Thomas $^{23}$, Stephen Vinter ${ }^{24}$, Jason Wakelin-Smith ${ }^{24}$, Jan Welink $^{25}$, Jianing Zeng ${ }^{19}$ \& Shaolian Zhou ${ }^{26}$

\footnotetext{
*Author for correspondence: Tel.: +390691045277

f.garofolo@angelini.it
}

Author affiliations can be found at the end of this article.

The views expressed in this article are those of the authors and do not reflect official policy of the US FDA, EU EMA, Health Canada, UK MHRA, Austria AGES, Italy AIFA, Brazil ANVISA, Japan MHLW and WHO. No official endorsement by the FDA, EMA, Health Canada, MHRA, AGES, AIFA, ANVISA, MHLW or WHO is intended or should be inferred.

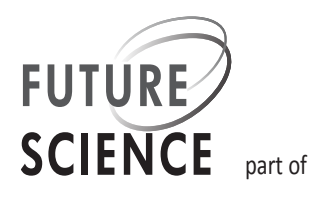




\section{Acronyms}

\begin{tabular}{|c|c|}
\hline Abbreviation & Definition \\
\hline ADA & Anti-drug antibody \\
\hline ADC & Antibody-drug conjugate \\
\hline AMED & $\begin{array}{l}\text { Japan Agency for Medical Research and } \\
\text { Development }\end{array}$ \\
\hline ANDA & Abbreviated New Drug Application \\
\hline API & Active Pharmaceutical Ingredient \\
\hline BA & Bioavailability \\
\hline BCS & Biopharmaceutics Classification System \\
\hline BE & Bioequivalence \\
\hline BLA & Biologics License Applications \\
\hline BMV & Bioanalytical method validation \\
\hline CDR & Complementarity Determining Regions \\
\hline CRO & Contract research organization \\
\hline CTA & Clinical Trial Application \\
\hline ELISA & Enzyme-Linked Immunosorbent Assay \\
\hline ELN & Electronic Laboratory Notebook \\
\hline GCP & Good Clinical Practice \\
\hline GCLP & Good Clinical Laboratory Practice \\
\hline GLP & Good Laboratory Practices \\
\hline HRMS & High Resolution Mass Spectrometry \\
\hline IA & Immunoaffinity \\
\hline IND & Investigational New Drug \\
\hline LBA & Ligand Binding Assay \\
\hline LCMS & Liquid chromatography mass spectrometry \\
\hline LOD & Lower Limit of Detection \\
\hline LLOQ & Lower limit of quantitation \\
\hline LIMS & Laboratory Information Management System \\
\hline mAb & Monoclonal antibody \\
\hline MRD & Minimum Required Dilution \\
\hline MRM & Multiple Reaction Monitoring \\
\hline NDS & New Drug Submission \\
\hline PBS & Phosphate-Buffered Saline \\
\hline PD & Pharmacodynamic \\
\hline PK & Pharmacokinetic \\
\hline $\operatorname{PrD}$ & Proteolytic Digestion \\
\hline PTM & Post-translational modifications \\
\hline QA & Quality Assurance \\
\hline QC & Quality control \\
\hline SIL & Stable Isotope Labeled \\
\hline SISCAPA & $\begin{array}{l}\text { Stable Isotope Standard Capture with Anti-Peptide } \\
\text { Antibodies }\end{array}$ \\
\hline SOP & Standard Operating Procedure \\
\hline WRIB & Workshop on Recent Issues in Bioanalysis \\
\hline
\end{tabular}

lin-Smith (UK MHRA), Mr Stephen Vinter (UK MHRA), Dr Fabrizio Galliccia (Italy AIFA), Mr Gustavo Mendes Lima Santos (Brazil ANVISA), Dr Mark Bustard (Health Canada), Dr Laurent Cocea (Health Canada), Dr Akiko Ishii-Watabe (Japan MHLWNIHS), Dr Yoshiro Saito (Japan MHLW-NIHS) and Ms Stephanie Croft (WHO).

The entire workshop was designed to complete the drafting of this White Paper based on the daily working dinners and open panel discussions among the lecturers, regulators and attendees. Each core workshop day covered a wide-range of bioanalytical, biomarker and immunogenicity topics requested by members of the community, and included lectures from industry opinion leaders and regulatory representatives.

As with prior WRIB editions [1-12], a significant number of topics were addressed during the workshop and condensed into a series of relevant recommendations. In this current White Paper, the exchanges, consensus and resulting recommendations on 32 recent issues ('hot' topics) in bioanalysis, biomarkers and immunogenicity are presented. These 32 topics are distributed across the following areas:

Small molecules, peptides and small molecule biomarkers by LCMS:

- Method development challenges in bioanalysis (six topics);

- Bioanalytical regulatory challenges (five topics);

- Hybrid LBA/LCMS for biotherapeutics, biomarkers and immunogenicity:

- Biomarker and immunogenicity assays (four topics);

$=\mathrm{PK}$ assays (four topics);

- Large molecules by LBA and cell-based assays:

- Immunogenicity (five topics);

- Biomarkers (four topics);

= PK assays (four topics).

In addition to the recommendations on the aforementioned topics, an additional section has been provided in the current White Paper that specifically focuses on key inputs from regulatory agencies.

Due to its length, the 2016 edition of this comprehensive White Paper has been divided into three parts for editorial reasons. This publication (Part 2) covers the recommendations for Hybrid LBA/LCMS and Regulatory Agencies' Inputs. Part 1 (Small Molecule, Peptides and Small Molecules Biomarkers using 
LCMS) and Part 3 (Large Molecule Bioanalysis using LBA/Cell-based Assays, Biomarkers and Immunogenicity) are published in volume 8 of Bioanalysis, issues 22 and 23 (2016), respectively.

\section{Discussion Topics}

\section{Biomarkers \& immunogenicity assays}

Biomarkers in solid tissues: benefits of hybrid LBA/LCMS \& specific sample preparation techniques designed to minimize interferences With hybrid LBA/LCMS or LCMS only methods, what are the best practices for sample processing, weighing of tissue, homogenization techniques, and pulverization versus sectioning of different tissue types? What are the best approaches for stability evaluations in prepared lysate, buffer selection, and storage conditions? What are the best practices for recovery and matrix effects, dilution linearity, sequential IA, and impact of lipids? What is the industry best practice in harvesting/freezing samples to preserve protein biomarker integrity, sample sourcing and normalization?

\section{Successful validation of sensitive confirmatory} protein biomarker assays by hybrid LBA/LCMS: practical recommendations to meet regulatory requirements

What is the importance of the use of SIL standards for hybrid LBA/LCMS biomarker assay validation versus other approaches? In cases when a surrogate tryptic peptide is used to quantitate a full-length protein analyte, what are the differences/caveats for using a full-length labeled protein as an internal standard compared to a winged peptide (with tryptic sites flanked by a few amino acids) compared to a fully tryptic labeled peptide? Have any comparisons been made to quantitate the same protein using all three approaches mentioned above? How well do full-length recombinant labeled proteins mimic/control for endogenous analytes? What are some criteria (in addition to correlation, BlandAltman plots) that can be used to determine whether a hybrid LBA/LCMS assay and LBA assay can be used interchangeably to get to the same conclusions? Is multiplexing better? What are some of the benefits/caveats compared to multiplexed hybrid LBA/LCMS assays? Would a full validation be warranted if one wanted to go to a 'uniplex' hybrid LBA/LCMS assay with one of the analytes from the multiplex assay?

\section{Latest advancements for improving drug}

tolerance \& specificity by hybrid LBA/LCMS

Can LCMS be used as an alternate platform for ADA analysis? Can a LCMS-ADA assay be used for screen-

\section{Key Terms}

Immunogenicity: The ability of a substance, including biotherapeutic substances, to stimulate an immune response in animals and humans.

Endogenous Substance: A molecule, protein or substance that originates from the biological matrix.

Catabolism: Formation of secondary molecules derived from complex molecules in living organisms.

Isotype: Antibody class and subclass of a given species.

Microsampling: Sampling of very small sample volumes $(\mu l)$ from animals and humans to assess drug and chemical exposure in biological matrix.

ing, titration, or both? How are LCMS-ADA assays validated? Are they similar to ADA immunoassays? What are the pros and cons of using a species-specific ADA positive control for validation? Is it important to determine cut-points for LCMS? Can LLOQ or LOD be used instead? Can LCMS-ADA assay improve drug tolerance of the assay?

Recent progress in multiplexing ADA by hybrid LBA/LCMS for antibody screening, titering \& isotyping

How do you establish cut-points when background $\operatorname{IgG}, \operatorname{IgM}, \operatorname{Ig} \mathrm{A}$ or $\operatorname{IgD}$ levels are below the detection limits due to clean background? What does the assay sensitivity mean when it is established with a positive control, especially if the positive control is not a humanized antibody? Does determination of the absolute amount of ADA provide any additional value in comparison to a traditional ADA immunoassay approach? Can LCMS be used to determine ADAs in the presence of high circulating concentrations of drug to overcome the issue of drug tolerance? Can LCMS be used for immunoglobulin allotyping/isotyping by specific immunocapture: IgG, IgM and other immunoglobulin subclasses and/or by specific signature tryptic peptides as a surrogate for the measurement of intact ADA? More work has been published on the use of LCMS for ADA assays confirming the possibility of using LCMS for immunogenicity studies. Was this work submitted to Regulatory Agencies? If yes, did it receive any questions from Regulatory Agencies?

\section{PK assays}

Use of hybrid LBA/LCMS technology compared to LBA alone to overcome the PK, biomarker \& immunogenicity assay challenges of novel biotherapeutic modalities

With tight timelines, it is difficult to wait until LBAs do not meet assay expectations to develop a hybrid 
LBA/LCMS method. Do bioanalytical scientists conduct LBA and hybrid assay development in parallel or in series, if one approach is not working? What is the best/recommended approach for method development to save time? Do scientists have criteria (perhaps a decision tree) that determine when to develop a LBA and when to develop a hybrid assay? Should we start with a hybrid assay by default? Overall, can hybrid LBA/ LCMS technology, compared to LBA alone, overcome the PK, PD, and ADA assay challenges of novel biotherapeutic modalities? What considerations and recommendations can we include to take advantage of adding LCMS to the traditional LBA approach as a starting strategy when developing assays for novel biotherapeutic modalities? Is it advantageous to perform a comparative evaluation of $\mathrm{PK}, \mathrm{PD}$ and $\mathrm{ADA}$ to detect common interferences?

Strategies to avoid ADA interferences in PK assays by hybrid LBA/LCMS

Can a direct LCMS assay (reagent free i.e. total digest) be used in combination with ELISA to better understand the PK of biologics in the presence of ADA? Are other groups performing free/total/bound measurements for biologics? What platform(s) are used? What information is gained from this? Can the high specificity of LCMS eliminate interferences and discriminate among isobaric interferences, modifications, substitutions, unique signature peptides not present on ADA? Is it possible to use harsher conditions than in LBA for ADA dissociations like alkaline, acid, Guanidine combo treatments?

Progress and fine-tuning of validation criteria for hybrid LBA/LCMS PK methods: building on previous recommendations

What is the best approach to test clinical hybrid assays for ADA resistance, especially where humans have not yet been dosed? What is the best solid state method for hybrid assays? If using beads, is on-bead or offbead digestion the best approach? Why? Should IA capture efficiency be routinely measured when Hybrid LBA/LCMS methods are validated? What is the best approach used by the industry to overcome problems with matrix effect determination for Hybrid LBA/ LCMS methods? Is there any progress from previous recommendations on recovery, digestion efficiency, protein assay stability and critical reagent evaluations?

Evaluation \& impact of mAb biotransformation in vivo on PK assay accuracy in non-clinical \& clinical studies

How widely is biotherapeutic biotransformation characterized in the industry? What is the best approach for biotherapeutic biotransformation characterization: top down versus bottom up versus integrated approaches? Why is it important to understand biotherapeutic biotransformation? What is the impact of biotherapeutic biotransformation on PK assays, safety, and efficacy? Is the use of LCMS' or HRMS' high specificity attribute the best approach to identify, characterize and quantify biotherapeutic biotransformations? How is the quantification of minor modified biotherapeutics in the presence of a large amount of unmodified entities handled?

\section{Discussions, consensus \& conclusions}

\section{Biomarkers \& immunogenicity assays}

\section{Biomarkers in solid tissues: benefits of hybrid} LBA/LCMS \& specific sample preparation techniques designed to minimize interferences

The expansion of new biotherapeutic modalities has increased the use of PK/PD modeling and system pharmacology. This has subsequently increased the demand from bioanalytical and biomarker groups with respect to quantitative measurements of biologics and biomarkers in both biological fluids and tissues. Tissue measurements come with increased scientific challenges compared to those in serum and/or plasma. The challenges come from many factors, such as procuring and handling the tissue sample, recovery of the analyte of interest and properly normalizing the target measurement of interest. However, quantitative tissue measurements, at the site of pharmacological action, can be critical as they may provide information to scientists about target validation, target coverage for dose projections, occupancy (total/bound), precision medicine for patient stratification, normal versus disease for target or biomarker measurements, membrane bound or intracellular proteins and, finally, drug disposition.

The use of Hybrid LBA/LCMS approaches offers an effective way to meet the requirements of sensitivity and cleanup associated with analyte measurement in solid tissue. However, best practice is strongly dependent on the analyte under investigation and intended use of the data. Hybrid LBA/LCMS methods are primarily used in discovery bioanalysis or for exploratory purposes since standardization and recovery tracking remains one of the major hurdles. In order to reduce potential interference from residual blood, in particular in PK analysis, it is recommended to wash the tissue sample using PBS upon tissue harvest and immediately flash freeze the sample in liquid nitrogen to prevent proteolysis. For exploratory, non-clinical studies, homogenization in acid at the collection site may be needed to better control the process, but obviously, this needs to be tested during assay development. 
When the intent is to perform a single measurement representative of drug distribution in the entire tissue sample, heterogeneity of the target protein can be a challenge. Hence, pulverizing and homogenizing the tissue is recommended to get a representative overall measurement. It was agreed that in this situation consideration should be given to the stability of the analyte at elevated temperatures, since heat may be generated during the homogenization process. When possible, samples should be kept at low temperatures during the homogenization process. Typically, a stainless steel or similar bead-based approach performed directly in vials is appropriate. It should be noted that different beads are available for different tissues. To properly weigh the tissue, it is recommended that tubes be pre-weighed with lysis buffer containing protease inhibitor, and then weighed following sample addition. It is generally preferred by PK/PD scientists for protein concentrations to be normalized against tissue weight rather than protein content. Following pulverization/homogenization, the tissue is removed and can be reprocessed using additional buffer if there is any concern that all the analyte may not have been extracted. If less than $5-10 \%$ is left in the second extraction, there is no need to repeat the process.

It was agreed among the scientists that currently there are limited options to improve analyte recovery from tissues, especially when combined with IA sample processing. Lipids can often cause poor protein recovery especially for protein biomarkers. Therefore, assessing the effect of lipids on the recovery of the protein analyte is recommended; a lipid clearing solution may be used. The overall consensus is that dilution typically increases recovery, thus samples should be diluted as much as possible in the homogenate lysate to maximize recovery and minimize interference. However, dilution may affect the measurement of certain types of analyte forms: such as free and bound estimations.

Acceptable stability parameters of the analyte need to be defined a priori, depending on the sample collection process and the purpose of the data. Some attendees stated that it is difficult to test analyte stability in tissues directly, so it was recommended to bank homogenate lysate and test it over time. Different buffers and different storage conditions should be tested on the homogenate (e.g., freeze/thaw a lysate pool) to help assess if there are problems with stability. Commercial lysis buffers with protease inhibitors generally work well, and cryopreservatives (e.g., glycerol) can be considered. Furthermore, it was decided that it is not necessary to perform incurred sample stability. Instead, lysates should be banked in order to possibly look at additional protein targets. Finally, careful consideration should be put into tissue sourcing. Attendees felt that the quality of commercial tissue sources, while easy to obtain, can be uncertain due to the uncertain nature of collection and handling. Therefore it may be preferable to obtain tissues via collaboration with trusted academic sites and hospitals where possible.

\section{Successful validation of sensitive confirmatory} protein biomarker assays by hybrid LBA/LCMS: practical recommendations to meet regulatory requirements

LBA has historically been the method of choice for quantitating proteins and peptides. However, inconsistent specificity and lack of reproducibility among products are some of the challenges of this approach when using commercial kits [13,14]. Hybrid LBA/LCMS assays are increasingly used in addition to LBA because these are highly selective methods to quantify proteins and peptides in complex matrices therefore can provide confidence in measuring analytes of interest. Users of these hybrid LBA/LCMS assays indicate this new platform to be reproducible, robust, and specific for biomarker measurements. The addition of an IA step increases the sensitivity of the assay in order to measure very low levels of proteins in samples. These assay platforms can provide a 10-fold increase in sensitivity, which can improve detection significantly [15]. Nevertheless, factors such as cost, sample volume requirements, assay throughput, and ease of implementation continue to be limiting factors to consider when deciding on the assay platform.

One of the cornerstones of a robust LCMS method is the use of an appropriate internal standard. Industry best practice is to use a SIL internal standard if available. This is equally important for Hybrid LBA/LCMS assays, and in cases where a surrogate tryptic peptide is used to quantify a full-length protein analyte, scientists have the option of using a full-length SIL protein, a winged SIL peptide where the tryptic peptide of interest is flanked by extra amino acids, or a labeled tryptic peptide of the exact length. If a labeled protein is available, then it is the best choice and if possible to use the same cell line as was used to produce the recombinant protein to maintain constant PTMs. However, industry experience indicates there is no determinable advantage in using a full-length protein versus a winged peptide; any differences encountered were likely due to other issues within the assay even if, in theory, having a full-length protein internal standard can be very useful when IA recovery is in question. Differences were observed when using a labeled protein versus a peptide only when severe solubility issues were present (i.e., membrane or PEGylated proteins). SISCAPA was considered an option. On-column capture should be used in this case. In conclusion, the 
overall recommendation is that as long as validation tests demonstrate that the assay is reproducible and robust, there was no preference for the type of internal standard used. Moreover, when assaying endogenous analytes, using a full-length recombinant labeled protein may be impacted by differential expressed protein isoforms and PTMs. Lot-to-lot variability of recombinant proteins is a significant challenge, and should be mitigated by minimizing the number of lots used, performing bridging assays and ensuring robust reagent characterization.

To determine whether a Hybrid LBA/LCMS assay and LBA assay can be used interchangeably to get to the same study conclusions, correlation and BlandAltman plots are still the recommended criteria. It is difficult to absolutely match historical data using different qualitative assessments. When moving to a uniplex hybrid LBA/LCMS assay from an already validated multiplex assay, it was agreed that some crossvalidation experiments were minimally recommended to assess comparability of results.

\section{Latest advancements for improving drug} tolerance \& specificity by hybrid LBA/LCMS

Therapeutic proteins and peptides have the potential to elicit immune responses resulting in ADAs that can pose problems for both patient safety and product efficacy. During drug development, immunogenicity is usually examined using a risk-based approach along with specific strategies for developing 'fit-for-purpose' bioanalytical approaches. LBAs are the most widely used platforms for ADA detection and characterization because of their high sensitivity and throughput. During the past decade, LCMS and Hybrid LBA/LCMS methods have been demonstrated to be successful techniques for the quantitation of biotherapeutics for PK assays and protein biomarkers in biological matrices, mainly owing to their high specificity, selectivity, multiplexing capability and wide dynamic range. More recently, Hybrid LBA/LCMS methodologies have been developed for simultaneous semi-quantitation and isotyping of the ADA in human and animal plasma or serum. ADAs are captured by biotinylated drug or the drug-ADA complex is captured by an anti-drug antibody. ADAs are then eluted and subjected to trypsin digestion followed by LCMS detection of specific peptides for each antibody isotype. The relative concentration of each ADA isotype in the extraction buffer is quantified using a calibration curve which is generated from reference materials of each ADA isotype prepared in the extraction buffer.

The proof-of-concept of this hybrid methodology is demonstrated by detecting pre-existing ADA in human plasma [16]. Either biotinylated drug or biotinylated
ADA could be used as the immunocapture reagent, each with its own merits and shortcomings. Biotinylated drug can readily capture ADA, but drug interference could be an issue if drug levels in the samples are high. On the other hand, immunocapture using an ADA eliminates the drug tolerance issue which is often associated with immunoassays, providing that the ADA is able to capture the drug-ADA complex in addition to free drug. With this method, unique peptides from each ADA isotype/subclass can be identified and monitored by LCMS. ADA isotyping can be performed by the detection of isotype-unique peptides. An absolute ADA amount can be obtained however the result is only semi-quantitative since the polyclonal ADA response cannot be mimicked with calibrants and commercially available Ig isotypes are spiked only post-elution to create the calibration curve. Similar to traditional ADA LBA assays, cut-points at 95\% confidence interval can be established using undosed controls. Endogenous Ig interferences need to be reduced in order to improve assay sensitivity. Human positive ADA controls or the controls containing human Fc are recommended for more accurate estimation of the LLOQ/LOD. Protein A/G beads can also be used to capture total immunoglobulins and then the drug-specific surrogate peptides can be measured to reflect the amount of the immunoglobulins bound with the drug. This application is only suitable to the drug which does not have binding affinity to Protein A/G, such as Fab domain antibody or other non-Fc proteins.

It was agreed that, with further improvements and industry experience, Hybrid LBA/LCMS methodology could become a useful tool in immunogenicity assessments. At present, this methodology is used for experimental purposes and not used in regulatory data acquisition. It can be easily implemented in bioanalytical lab settings for routine ADA isotyping and semiquantitation. The key issue is the reliability of immunocapture step. As ADA levels measured by Hybrid LBA/LCMS represent absolute amounts relative to the positive control used, one can compare ADA isotype levels between samples, between studies, as well as between different biotherapeutics, providing that consistency in positive controls is achieved to determine recovery. A database of such information could be gradually built and provide valuable insight to better understand immunogenicity of biotherapeutics.

It was also agreed that Hybrid LBA/LCMS ADA assays could become an alternative for screening, isotyping, and titration without a need for confirmatory assays due to better specificity (lower false positives). In cases where there is interference from the drug itself, there could be benefits for a confirmatory assay, but this is assay and positive control dependent 
and more information is needed prior to widespread adoption. Each $\operatorname{IgG}$ response is different from patient to patient, so it is critical to compare post-dose samples to the same patient's pre-dose sample. In the case where Hybrid LBA/LCMS could be used for screening assays, a separate titration may not be required because the screening assay provides the relative concentration of ADA. However, the assay is only semi-quantitative because reference standards are not spiked into the matrix. Commercial IgG or IgM, not the actual ADA, is used to run the calibration curve. The selected surrogate peptide may be significantly different from the actual ADAs, even if the same isotype but difference species.

Neither the 2009 draft regulatory guidance for ADA assays [17] nor its 2016 revised version [18] discuss the use of Hybrid LBA/LCMS for immunogenicity assessment. Therefore, it is recommended that the requirements for the validation of Hybrid LBA/LCMS ADA assays be leveraged from the best practices and lessons learned from LBA ADA assays. In addition, because of the absence of regulatory guidelines regarding validation of Hybrid LBA/LCMS ADA assays it is recommended that sponsors contact the Regulatory Agencies and discuss assay validation plans with them prior to conduction. Currently, using a species-specific ADA positive control for validations can help establish detection limit, and is essential for surrogate peptide selection in Hybrid LBA/LCMS assays. Usually due to availability reasons, positive controls are often monoclonal whereas an ADA response is polyclonal. However, positive controls cannot represent all endogenous ADAs, introducing limits to the method validation. This is the same as for the LBA ADA assays. Cut-points are important statistically when a broad population is evaluated, so it is likely both cut-points and LLOQ/LOD are needed.

\section{Recent progress in multiplexing ADA by hybrid LBA/LCMS for antibody screening, titering \& isotyping}

Currently, LBAs are widely used platforms for evaluating the immunogenicity of protein therapeutics, and a tiered approach is used to screen, confirm and titer the ADA present in patient samples. However, drug interference in the assay can prevent low level and low affinity ADAs from being detected. When it comes to isotyping of ADAs, although new technologies are being developed with LBAs, these technologies have not been broadly utilized in the pharmaceutical industry due to their complexity, as well as the infrequent and unclear need for isotyping. It was agreed that Hybrid LBA/ LCMS assays can be used for semi-quantitative measurement of ADAs through either direct measurement of $\operatorname{IgGs}$, IgMs, $\operatorname{IgAs}$, IgEs and $\mathrm{IgDs}$ and their respective subclasses bound to the drug, or indirect measurement of the drug bound to ADAs. However, the use of Hybrid LBA/LCMS assays to determine the relative amount of ADA does not show additional value in comparison to a traditional ADA LBA approach. There are still limitations to using LCMS for very low levels or low affinities of ADA due to lower sensitivity than LBAs. Additionally, it is not clear why isytoping would add value in most cases. Sensitivity for a Hybrid LBA/LCMS assay will be established using cut-points when the positive control is not a same species antibody because different surrogate peptides are measured.

There has been recent progress in multiplexing ADA measurement and isotyping immunoglobulin subtypes by Hybrid LBA/LCMS [16]. This technology provides a way of overcoming the drug tolerance issue as long as adequate sensitivity can be established. However, background issues are still an overall challenge. More work has been published on the use of Hybrid LBA/ LCMS for ADA assays confirming the possibility of using LCMS for immunogenicity studies and providing an alternative to the traditional tiered approach that uses three separate assays. However, regulatory agencies report that they have not yet reviewed any submitted ADA data using Hybrid LBA/LCMS at this time. Despite being a young application for Hybrid LBA/LCMS, multiplexing isoyping of ADAs is already used as a proof-of-concept for ADA determination but as industry and regulatory experience increase more of these assays are expected.

\section{PK assays}

Use of hybrid LBA/LCMS technology compared to LBA alone to overcome the PK, biomarker $\&$ immunogenicity assay challenges of novel biotherapeutic modalities

Novel biotherapeutic modalities include fusion proteins, bispecific antibodies, ADCs, and other emerging new entities. Developing assays for these modalities involves overcoming additional challenges to those already evident with monoclonal antibody therapeutics. The challenges, including assay interference from endogenous protein drug targets (usually a soluble target) or ADAs in PK assays, drug or target in ADA assays and target or binding partners for PD assays, must be overcome to develop an accurate and robust method. It was discussed whether a comparative evaluation of PK, PD and ADA data could help detect common interferences. It is recommended that the biology would first have to be examined to decide if common interferences are likely. Further, the interferences would be different based on the platform, limiting the practicality of this 
approach. The procurement of the necessary reagents may also be a challenge for the novel therapeutics. There were many advantages acknowledged for combining LCMS with traditional LBA to create a Hybrid LBA/LCMS approach. LCMS can better characterize exactly what is being measured, improve the understanding of the analyte as well as the biology involved and also detect in vivo modifications (e.g., clipping of biotherapeutics with an endogenous counterpart). Method development is generally faster compared to traditional LBA, and comes with improved selectivity and less stringent reagent requirements. It is worthwhile to mention here that Hybrid LBA/LCMS assays can use reagents that do not work in LBA assays due to better selectivity of the mass spectrometers. It is also generally more resistant to interference due primarily to the specificity offered by the detection step and the use of only one antibody for capture. However, it should be noted that the Hybrid LBA/LCMS and LBA may not measure the same species, depending on the assay formats. Therefore, in some cases it is desirable to use both assays for bioanalysis in order to get a better understanding of PK/PD. On the other hand, caution should be exercised when comparing results from the two assays.

Overall, it was agreed that presently LBAs are often still used as the default approach largely due to the confidence resulting from a successful history with this technology, regulatory acceptance and existing in-house and CRO capabilities. Hybrid LBA/LCMS assays can also be used as the main PK assay or when issues like those discussed above arise. For a more proactive approach, it is recommended that scientists should begin developing LBA and hybrid methods in parallel with a clear path as to when to continue using one methodology based on initial result generation. This approach will work if a clear strategy is in place, allowing scientists to make an informed decision based on the pros and cons of both methodologies, as well as taking into account the phase and the biology of the drug that it would be used for. The current reality is that most scientists work in series due to financial and resource limitations. Furthermore, using a hybrid method limits the ability to transfer the method to another lab such as a CRO, who may not have that platform capability. It was agreed that Hybrid LBA/ LCMS is a possible orthogonal method to LBA for PK and PD assays [19,20].

Strategies to avoid ADA interferences in PK assays by hybrid LBA/LCMS

The ability to accurately quantify total concentrations of biopharmaceutical therapeutics in the presence of ADAs is sometimes required to understand the safety and/or efficacy of these molecules in both preclinical and clinical studies. These studies are traditionally supported using LBAs which require binding of the therapeutic to a capture reagent (e.g., antigen capture or anti-idiotypic capture) and a detection reagent (e.g., Anti-Human IgG antibody or anti-idiotypic antibody) for quantitation. However, the presence of an ADA may compete with the binding of the therapeutic to the capture and/or detection reagent, thus preventing measurement and masking the presence of the circulating molecule. This interference can thereby have a damaging impact on safety and/ or efficacy interpretations [21]. Recommendations have previously been made for assessing the impact of immunogenicity on PK assays $[9,12,22]$. Since then, an orthogonal approach like LCMS has emerged as an alternative tool to better understand the PK of biologics in the presence of ADA. It was agreed that the high selectivity of LCMS can minimize interferences and discriminate among isobaric interferences, modifications, substitutions, and unique signature peptides not present on an ADA. However, bioanalytical method characterization of ADA tolerance is imperative. Some traditional ways for solving ADA tolerance issues include acid dissociation, acid capture elution (ACE), biotinylated or passive adsorption, and increasing minimum required dilutions. When traditional LBA options do not solve these method issues, it is recommended that LCMS or Hybrid LBA/LCMS methods with harsher conditions than those typically used for LBA (e.g., alkaline, acid or guanidine combo treatments) could be used to achieve ADA dissociations. The appropriate platform for PK/PD endpoint assessments should be based on a biology driven strategy with consideration given towards the collective endpoints for interpretation.

\section{Progress \& fine-tuning of validation criteria} for hybrid LBA/LCMS PK methods: building on previous recommendations

Much attention has been paid in the last several years on the validation of protein bioanalytical methods that quantify surrogate peptides via LCMS after proteolytic digestions [6,8,11]. An enhancement of these LCMS methods increasing in prevalence is the Hybrid LBA/ LCMS approach. This approach utilizes affinity capture reagents to enrich the protein drug from matrix prior to digestion. These reagents can range from less specific affinity reagents such as protein A to highly specific immune-affinity capture reagents such as antiidiotypic monoclonal antibodies or drug target. Techniques for coupling these affinity capture methods to the LCMS normally employ solid state methods using micro particles such as magnetic or sepharose beads 
coated with streptavidin that binds to biotinylated reagents. The result is an increase in method sensitivity of up to 100 times allowing such assays to be validated and applied to a number of clinical and non-clinical studies with good success [11].

It was agreed that beads were the best solid state method for Hybrid LBA/LCMS assays, although the choice is assay dependent. On-bead digestion is recommended because of its ease and lack of extra steps when compared to off-bead digestion, although it was acknowledged that the off-bead approach is ideal because it gives the most complete digestion and is subject to less interference from non-specifically bound materials found on the beads. For many applications on-bead digestion is often sufficient for the purpose of the assay.

Along with the enhanced performance of Hybrid LBA/LCMS methods, some of the potential complications associated with LBA may become apparent. These include possible effects due to ADAs, circulating ligands, or specific interferences in patient populations and/or dosed subjects. Since these factors can directly impact quantitation and recovery, they must be evaluated carefully during method validation and monitored during sample analysis. Hence, it is recommended for Hybrid LBA/LCMS methods to a) study selectivity in both pooled control and pre-dose patient matrices; b) examine the effects of specific interfering ligands, such as soluble targets, at expected physiological concentrations upon drug quantitation in spiked QC samples; and c) examine the impact of added ADAs of known high titer, if available upon quantitation, in spiked QC samples. The best approach to test clinical Hybrid LBA/LCMS assays for ADA resistance, especially in drug naïve patients, is to use pre-existing $\mathrm{ADAs}$, animal ADAs as positive controls.

It is recommended that recovery and digestion efficiency in validation are examined at low, mid and high QC concentrations. Special validation experiments designed to specifically measure affinity capture recovery should be tested with both spiked control and patient pre-dose samples to determine whether there is any effect of the patient matrix on immunocapture efficiency. The recovery results provide information on selectivity and matrix effect, which is a known difficulty when using mass spectrometry as a detection tool. Testing patient pre-dose samples as naive and spiked at the LLOQ concentration is the recommended method for testing matrix effect and selectivity.

It is suggested that parallelism experiments be performed during validation on incurred samples by diluting with disease patient, pre-dose, and control matrices, followed by quantification. The results are then compared to those diluted in buffer.
Finally, it was agreed that the issue of reagent management requires considerations similar to LBA reagents. Hence, reagent stability, suitability, and lotto-lot comparability can often be evaluated on a fit-forpurpose basis. Many of these reagent management concerns can be handled by working in close collaboration with the reagent group of the organization due to their experience and extensive use of methodology to verify, characterize and maintain these critical reagents.

\section{Evaluation \& impact of $\mathrm{mAb}$ biotransformations} in vivo on PK assay accuracy in non-clinical \& clinical studies

Drug development is a slow process with a high failure rate, especially when new drug modalities are being introduced. At the same time, biotherapeutics are becoming even more structurally complex, with formats including bispecifics, fusion proteins, antibody fragments and cyclic peptides. Analytical methods to understand the fate of biotherapeutics in vivo, including structural stability or catabolism, have the potential to provide valuable information during drug development and may help speed up the process in getting drugs to patients. Historically, such a depth of structural information has not been available for biotherapeutic development. However more recently, mass spectrometry-based approaches have been used to explore large molecule in vivo catabolism $[23,24]$ and is considered the best approach to identify, characterize and potentially quantify biotherapeutic biotransformations such as antibody-drug conjugates. Hybrid LBA/LCMS approaches developed for ADCs can be extended to mAbs and other modalities to identify biotransformations including deamidation, clipping and isomerization [11]. Such biotransformations in the proximity of the CDR target binding or Fc effector function binding regions of a mAb biotherapeutic has the potential to alter the in vivo pharmacology, affecting efficacy, safety, and PD evaluations. Biotransformations in the $\mathrm{CDR}$ region for $\mathrm{mAbs}$ also has the potential to alter binding to assay reagents in LBA or Hybrid LBA/LCMS PK assays and yield anomalous PK measurements. Understanding biotherapeutic biotransformations, therefore, will help scientists determine the impact on efficacy, safety, PD, and PK, as well as help to correlate in vitro monitoring. Traditionally, characterization of biotherapeutic in vivo catabolism is very limited and only done when needed. Top-down or middle-down approaches, which analyse the intact biotherapeutic or its sub-units (e.g., light chains and heavy chains of the $\mathrm{mAb}$ ) via immunoaffinity capture and mass analysis. Another option is a bottom-up approach, where MRM or HRMS full scan analyses are used to target specific peptides or an open 
proteomics discovery approach to identify biotransformations. However, it was agreed that an integrated approach would be the preferred option. In the case where it is necessary to quantify minor biotransformed biotherapeutics in the presence of a large amount of unmodified biotherapeutics, it is recommended to follow the MIST guidance for small molecules [25] present in quantities of more than $10 \%$. The dynamic range of the method should allow for proper analysis. For quantities below $10 \%$, the minor biotherapeutic is unlikely to be relevant, depending on the importance of biotransformation.

\section{Key input from regulatory agencies}

The 10th WRIB continued with its long standing contributions from several international regulatory agencies, including the US FDA, EU EMA, Health Canada, UK MHRA, Austria AGES, Italy AIFA, Brazil ANVISA, Japan MHLW and WHO. These agencies shared their views on multiple topics of interest for the global bioanalytical community attending this event, to provide clarification on unresolved issues or expectations. Regulators provided input on a variety of topics across all three core days, including:

- Microsampling ICH

- Extract stability

- Batch acceptance criteria

- Electronic data integrity

- Endogenous compounds

- Validation of biologics and biosimilars

- Immunogenicity issues

- Inspection programs, observations and new guidelines

An update on the recently released draft S3A Q\&A document focusing on microsampling for toxicokinetic studies [26] was provided by a representative from Japan MHLW, who is the rapporteur of the ICH implementation working group that prepared the document. The document contains questions addressing microsampling covering scope, application, effect on safety and issues regarding bioanalytical methods. It was started in early 2015 and took approximately a year to complete and was released for comments. Consultation was open until September 2016 on the ICH web site, and it is anticipated that the final version will be adopted by spring or summer of 2017 .

The subject of extract stability was brought back to the table by the US FDA, following up on the original discussion in the 2009 White Paper in Bioanalysis [1]. The Agency stated that there are still differences in industry practices with regards to the evaluation of extract stability. The US FDA representative reminded those present that the objective of the extract stability evaluation is to determine stability of the analyte(s) and internal standard(s) post-extraction. This is referred to as the post-preparative stability in the US FDA Guidance on Bioanalytical Method Validation [27]. Post-extraction storage of samples may be at room temperature or colder, and extract stability can address stability of the analyte following the resident time of the samples on the autosampler for the duration of the run. This is particularly relevant if the run is interrupted for some reason, and injection is continued; the analyte and IS stability should be preserved from the beginning of the run to the end of the run. Two procedures were presented. The first was to inject high and low QC stability samples along with a freshly prepared set of calibrators and analyze the QCs. The stability QC samples are then subjected to storage conditions for a determined period of time and reinjected using freshly spiked calibrators. Calculated concentrations are then compared to nominal values for determination of stability. Some advantages of this approach are that the use of ratios addresses possible differences in ionization or other sources of variability, and freshly prepared calibrators are used in case of similar degradation between analyte and IS. Some disadvantages to consider are that the fresh calibrators are not processed with the stability samples, introducing additional variability (e.g., extraction variability), and once the sealing mat is punctured for the stability QCs, there is a potential for evaporation. Another method was proposed, where the stability samples are extracted with a fresh set of calibrators and batch QCs. The curve and batch QCs are injected immediately, but the stability QCs are not injected with these. Instead, stability samples are immediately stored and injected on a future day (after a period of aging), but read against the originally injected curve. In this case, all samples are processed (extracted) together and there are no issues with evaporation, since the sealing mat of the stability samples remain unpierced. This method, however, assumes stable instrument response and spray conditions over the course of the experiment. Neither method was favored as the preferred strategy. However, the pros and cons of each approach should be considered when determining the most appropriate design of the experiment based on the method.

The US FDA also introduced the topic of batch acceptance. This is particularly pertinent when multiple 96-well plates are used, and each plate is processed individually but injected in sequence in one run. 
Regulators have come across data where batch criteria were applied across the multiple plates, as opposed to each plate individually. However, in cases such as this, where each plate was processed individually, the expectation is that there is at least a complete set of QC samples on each plate along with study samples. This way, once the instrumental run criteria pass, each batch can then be evaluated independently. This method provides the most information about the quality of the data generated and is consistent with good science, US FDA recommendations and the EU EMA guideline on BMV [28]. It is important that SOPs clearly define what constitutes a batch.

Austria AGES and US FDA addressed the increased focus by regulatory agencies on electronic data. Data integrity is of primary concern, thus inspectors are very vigilant in identifying signs of manipulation or falsification. Data integrity issues are not limited to e-records, electronic storage media and software, but also involve all information management systems used by a company. Appropriate data management, as well as maintenance of data integrity, are areas prone to risk and should have an adequate level of risk analysis. Poor data management can open the door for unpredictable malpractices. Electronic laboratory notebooks have benefitted many companies by improving efficiency and productivity, prolonging record longevity, allowing for efficient and global information sharing and retrieval, and by facilitating real time data review. Industry has been discussing the impact of their use for many years $[8,11,29]$. Regulators indicate that they are reviewing data from a variety of different ELN systems, as one size does not appear to fit all, resulting in many challenges occurring during site inspections. ELN formats, structure and granularity differ from site to site, forcing regulators to take time in learning the navigation of the system. Hyperlinked information in worksheets may be read-only or links may be broken, thereby causing delays during the inspection. This may result in extended waiting times for accessing all required information while waiting for site staff knowledgeable in the system. Furthermore, analytical data audits become challenging when different aspects of the analytical data are documented in different systems (e.g., old versus new, paper versus ELN). Therefore, the inspection scope has been expanding in order to comprehend the IT aspects of ELNs for assuring data integrity. Documentation of study data within an ELN should still follow all predicate rules. The individuals who carried out each aspect of study conduct should be clearly captured, as well as the date and time when the study events actually occurred, and not just when they were recorded. This is usually done via e-signatures and timestamps. Access must be available to the audit trail to determine what changes occurred and the individuals who made them. It should also be noted that efforts are made to inspect the ELN source files and not just PDF export files. It was recommended that any access granted to the inspectors should not affect the system performance. However, inspectors should still be able to completely reconstruct the study within the system. To quickly orient themselves with the ELN structure and possible links to other LIMS at the firm, regulators may request a system map. Finally, ELN SOPs should be in place to elaborate on topics such as the overall workflow and archiving, version control of forms and templates used, audit trails, the ELN review and approval process, and the roles and responsibilities within the ELN. Apart from ELN systems, all electronic data are within the scope of a regulatory inspection. Regulators still consistently note cases where there are no personnel with expertise in computer validation at the firm. Gaps in the proper validation of a computer system increase the risk of data integrity issues. The evaluation of risk must include assessments on the possibility of deleting or modifying data from outside the system, network storage of analytical and PK data without transparent access control, intermediate study results that flow prematurely via unauthorized access to the software, and ineffective QC and QA verification practices. Access to the software must be controlled and justified by the job description. The data flow must be clearly described in the processes such that the verification of batch results, data approval, release for PK and handoffs to pharmacokinetic scientists and QA are clearly tracked and documented. Any weaknesses of the quality system, data management and the use of computerized systems make it difficult for regulators to trust the position of management and results in significant suspicion about the reliability of trial data, thereby requiring further investigations during the inspection.

US FDA provides some guidance on the analysis of endogenous compounds [30-32], and some white papers have also provided recommendations on this topic [33,34]. Endogenous compounds can originate from the endocrine glands (e.g., glucagon, estrogens) or from dietary uptake (e.g., iron, omega 3 fatty acids). When designing a study for endogenous compounds, several points need consideration. First, the selection of the matrix should be appropriate. The PK characteristics of the drug substance should be considered in the selection of the matrix (e.g., selecting urine to assay potassium because potassium concentrations in the body are controlled largely by the kidneys through urinary excretion). The stability of the analyte in vivo is also important. Consideration for the use of low background level populations may reduce the need 
for baseline level corrections (e.g., post-menopausal women for estrogen studies). If corrections are needed, an evaluation of the characteristics of the baseline levels is beneficial (i.e. expected variations and magnitude of variations), as well as the methods that will be used to perform the correction (i.e. point-to-point, average of pre-dose values). Method validation of endogenous compounds includes some extra challenges. It is necessary to reliably determine the baseline level of the compound in the matrix of choice. If a surrogate matrix is used, additional validation data are needed to show equivalent performance of the analytical method between the surrogate and the subject matrix. QC samples prepared in authentic matrix should always be included during accuracy and precision. Stability in authentic matrix is recommended, even for rare matrices. Recovery between the analyte-free matrix and authentic matrix should be comparable and complete. Assay performance in multiple lots of surrogate and authentic matrix should be cross-validated to prove that there are no matrix effects. It was made clear that while the fit-for-purpose concept for the validation of biomarker assays is appropriate for different stages of drug development, for ANDAs, submission of a fully validated analytical method is expected.

US FDA shared their experience on the validation of immunogenicity assays for biologics and biosimilars. Indeed, these submissions have increased over the last several years as more pharmaceutical products of these types are being developed and marketed. As a result, regulatory experience with these types of submissions is more readily available. Cases exist where a low positive control concentration in an ADA assay was selected with an assay value much higher than the assay cut point and/or study sample values. This is concerning because there is no indication of precision at the low end of the assay range, resulting in uncertainty that lower ADA concentrations can be detected. Guidance [16,35] and literature [36] recommend selecting a low positive control concentration resulting in assay failure at a rate of $\sim 1 \%$. Non-reproducibility between Tier 1 and Tier 2 assays also raises concerns for the inspectors, because assay reliability is then uncertain and the potential for false negatives in the Tier 1 screening assay increases. There is a potential for misidentification of ADA positive samples and inaccurate assessment of the ADA response rate when samples are defined as negative during the neutralizing assay because they were less than the screening cut point even when they were confirmed positive and no further analysis is performed. Confirmatory cut-points should not be set too high, nor be variable; otherwise there is no assurance of assay specificity. Titer assay cut points are of equal concern when dilutions of patient samples for the Tier 3 assays are performed with normal matrix instead of patient matrix, due to e.g. unavailability. In these cases, reported titer is not indicative of the true titer, and there is a potential failure to err on the conservative side of titer reporting. Finally, using a LIMS system for immunogenicity assays brings risks for run identification errors, failure to monitor raw assay values of positive controls and for the deactivation of valid assay results. Immunogenicity data generated using LIMS systems should ensure that no acceptable data is deactivated and that there are pre-established criteria for reporting of final titer values and for governing sample repetition specific to the titer assay.

US FDA, Health Canada and Japan MHLW addressed some immunogenicity issues. Health Canada presented some typical issues that are seen during the review of immunogenicity data as part of marketing approval submissions. Currently, it is very difficult to determine the impact of drug immunogenicity on the benefit/risk profile of a biologic. Safety of the patients should be evaluated in the context of the particular disease being treated as well as by comparison to similar products on the market or in development, which may shed light on alleviating any safety issues. For example, adverse events related to immune reactions should be monitored within a meaningful timeframe, which can range from hours to several months after the last dose of treatment. Determining the impact of ADAs on efficacy is also a challenge. Some examples were provided where data was unable to show either the neutralizing or the non-neutralizing antibodies' impact on efficacy. Another case was shown where the long-term persistence of ADA was widely variable across the patients tested, and no conclusions could be drawn on the impact. Finally, the quality of the methods for detecting ADAs could be questionable. Methods were sometimes not adequately validated, especially with regards to specificity and sensitivity, and so data drawn from these methods were not considered reliable. In light of these challenges, Health Canada clarified that, at the CTA stage (equivalent to the IND step in the US), the apparent immunogenicity of the test drug alone is not considered a 'show stopper' as long as the risk/benefit profile of the drug is acceptable. Furthermore, at the NDS stage (equivalent to the BLA stage in the US), the agency's clinical decisions are based on the efficacy response and the benefit/risk profile of the drug, since, typically, study results conclude that no correlation between immunogenicity and efficacy/safety was found. Health Canada evaluates the role of immunogenicity in each submission on a case-by-case basis and collaborates with other regulatory agencies to develop a consistent approach. Japan MHLW indicated that they do not have specific guidelines for immunogenicity 
assessments. However, EU EMA and US FDA guidelines/guidance addressing the subject are good references to use for Japanese submissions, as the basic concepts are similar to the approach taken in Japan. The strategy for immunogenicity assessments, including the sampling schedule, depends largely on the clinical target product profile. In order to fill the gap of lack of guidance in Japan, an AMED research group is in the process of creating a document that includes some points to consider for ensuring the reliability of ADA assays. This document will be the basis for a future guideline for immunogenicity assessments of therapeutic proteins in Japan. Some considerations include cut point determinations, drug tolerance and biosimilars. Some additional questions regarding immunogenicity were posed to the regulatory panel. First, the situation was presented where some screening positives came in under the cut point in the confirmatory assay. This was considered acceptable because some false positives do occur and are expected, and the confirmatory assay is used to confirm this. Regulators agreed with this, however, their concern is raised when a significant portion of the samples demonstrate screening false positives. In this case, an investigation would be warranted. Regulators were also asked their opinion on using a raised titer cut point instead of a screening cut point. It was acknowledged that some scientists follow this practice, and it is supported in the literature, however, one should err on the side of caution when deciding to accept this approach.

Several agencies presented summaries of their inspection programs as well as some examples of their observations and new guidelines:

UK MHRA highlighted that although bioanalysis is often discussed under the guise of GLP regulations $[37,38]$, it is important to underline that clinical study samples must be analyzed under GCP [39]. Often, bioanalysts claim that since they only deal with bioanalytical samples and not clinical patients, GCP is less relevant. However, subjects have certain rights and these rights extend to the way that clinical samples are taken, processed, analyzed and reported. Guidance exists for laboratories that perform the analysis of clinical trial samples $[40,41]$ in order to capture the relevant portions of each set of practices. However, harmonization between agencies on the applicability of GCP (UK MHRA, US FDA via 21CFR320, Health Canada, Japan MHLW), GLP (ANVISA) or GCLP (WHO) to clinical bioanalysis is not consistent. That being said, regulators expect that the roles and responsibilities outlined in the GCP guidelines be followed. Training of staff working on clinical studies must be appropriate in education, experience and technical trainings received on the job.
UK MHRA continued its regulatory input by stating that staff involved in the analysis of samples from clinical trials must have an appropriate educational background, relevant experience and have received appropriate technical training relating to the work they undertake. GCP training commensurate with the employees' roles and responsibilities is required, and should be refreshed at appropriate intervals. Finally, unblinding of samples was discussed. The bioanalytical principal investigator first needs to consider whether there is an actual need to unblind the samples and what the consequences of unblinding for the trial might be. Any request to unblind must be approved by the sponsor. The process for unblinding should be outlined in an SOP and well documented including the rationale for the decision. Once the decision to unblind has been taken, control of the unblinded data is essential and consideration should be given to who needs to know and how the information will be communicated. Storage, transfer and retention of the unblind data also need to be considered. The clinical study report should include details of the need to unblind study samples and whether there is any perceived impact on the conduct of the trial.

Italy AIFA underlined that although a sponsor may transfer any or all of their trial-related duties and functions to a CRO, the ultimate responsibility for the quality and integrity of the study resides with the sponsor. Therefore, the sponsor as well as the applicant, if needed, should demonstrate the controls in place in order to guarantee the quality and integrity of the study data. For the clinical portion of the study, this is required via the monitoring function. If monitoring is extended to the bioanalytical portion of the study, qualification of the monitor in the specific field should be demonstrated and documented. Independent audits, performed by qualified people, are also an acceptable way to verify the bioanalytical phase of the study. In any case, laboratories should be included by the sponsors in their qualification programs of vendors/third parties.

WHO presented a list of case studies on clinical trial issues, violations and impact on bioanalytical data reliability. Most products submitted for WHO prequalification are generics and are therefore supported by clinical bioequivalence studies or biowaivers. WHO inspections have shown that data integrity is a worldwide issue and both the sponsors and the CRO are responsible. An increasing trend on the number of falsified study data occurrences have been seen and could be due to a number of reasons, including an increased rate of detection due to recent focus and training of inspectors in the area of data integrity. It was hypothesized that one of the motives 
for data manipulation could be that the sponsors believe that it is less onerous to falsify bioanalytical data than to reformulate a failing product. On the other hand, the CROs want to provide sponsors passing studies to avoid the sponsors seeking out other competing CROs. WHO, in collaboration with other agencies, are actively working to assure data integrity by increasing their focus on data appearing suspicious, by including inspectors with IT skills as part of inspection teams, as well as increasing awareness on the topic by the publication of guidance to industry $[42,43]$.

Brazil ANVISA presented some summaries of new technical notes that have been released since last year's WRIB conference. The first covers bioequivalence studies for generic products which contain vitamins above the daily intake and associated with APIs [44]. Bioequivalence to a Brazil ANVISA reference drug must be shown. A BCS biowaiver is not applicable. PK parameters of the unaltered vitamin in the appropriate matrix are required. Baseline values at specific intervals are required to establish a mean concentration per subject, which is then subtracted from the calculated concentrations. Vitamin intake from food must be controlled; a detailed menu must be submitted. The technical note regarding PK interactions for fixed dose combinations [45] is mandatory to follow when the efficacy and safety of the combination has not been documented in the literature or when one of the molecules is a new molecule in Brazil. Two BA studies would be needed comparing the combination dose to each individual Brazil ANVISA reference product. Regarding regulations for PK studies for biosimilars [46], the applicable LBA or LCMS validation criteria [47] is expected. Reporting must follow resolution RE 895/2003. It is recommended to conduct these types of studies in Brazil ANVISA certified CROs.

Health Canada advised attendees of three new documents that they had recently released. The first is a draft guidance document sent for consultation, which discusses the disclosure of confidential business information [48]. The final version of their Proposed Policy on Bioequivalence Standards for Highly Variable Drug Products [49] had also just been recently released as well as a guidance on Post-Notice of Compliance (NOC) Changes [50]. Following this update, the latest perspective on their notice on the Clarification of Bioanalytical Method Validation Procedures [51] was discussed. This notice was released by Health Canada in October 2015 with regards to the number of tubes used to perform matrix-based stability evaluations. Immediately following the notice, the agency began issuing deficiency letters asking for clarification, or in some cases, additional stability information based on the specifics of each case. The interpretation of the notice by the industry was not uniform, and deficiency letters were asking for different stability evaluations to be conducted depending on the details of the case, causing generalized confusion over the scope of the requests. The impact was wide-reaching and costly for applicants in both resources and time. In order to clarify the agency's position on this topic, an addendum to the notice was issued in March 2016 [52] and discussed at the conference. The notice applies to bioanalytical methods (primarily employing LCMS) for the measurement of small molecules in BE studies that are pivotal to the regulatory assessment of human therapeutic drug products in generic and innovator submissions. Health Canada's interpretation and expectations for all matrix-based stability experiments (i.e. long-term, freeze-thaw and bench top) is that a minimum of 3 tubes be stored and assessed at each QC concentration. Typically in the past, matrix-based stability experiments were reported using six values $(n=6)$, and it had been assumed that each value represented an individual tube. However, the agency came to understand that some companies were storing one single bulk tube, but then processing and misreporting the data as six replicates. Health Canada considers this a misrepresentation of the data, since sound scientific practice indicates that this modified approach should be reported as a single $(n=1)$ stability tube. The US FDA and EU EMA BMV guidance/guidelines also specify that a minimum of triplicate values must be reported. During the discussion the other regulators were in line with Health Canada's thinking and expectations. It should be noted, however, that there was considerable disagreement from attendees, who claim that their results show no impact from storing a single bulk tube or replicate tubes. In response, the agency noted that they were open to industry compiling data to support their position, but strongly cautioned that such data is specific to an ingredient and to conditions of the method, so that extrapolating across ingredients and methods would likely not be acceptable. Going forward, data submitted prior to the October 2015 notice will be evaluated on a caseby-case basis to determine if additional stability data will be requested. However, it is expected that all submission after October 2015 contain matrix stability performed using a minimum of three tubes per QC concentration.

\section{Recommendations}

Below is a summary of the recommendations made during the 10th WRIB. 


\section{Biomarkers \& immunogenicity assays}

1. It is recommended to wash solid tissue samples with PBS during sample collection to remove possible blood contamination and flash freeze as soon as possible. When heterogeneity of the analyte distribution is a concern, use of pulverization and homogenization techniques is recommended for the entire tissue sample to ensure a representative overall measurement. Lipids can often cause a poor recovery, therefore performing a correlation with lipid concentration and recovery of protein analyte is recommended using a commercially available lipid clearing solution. It was agreed that it is very challenging to test analyte stability in tissues, so it is suggested to test stability of homogenate lysate over time instead.

2. For protein biomarker assays by Hybrid LBA/ LCMS, as long as validation tests demonstrate that the assay is reproducible, there is no preference which type of labeled internal standard, whole protein, winged peptide or exact peptide, should be used. To determine whether a Hybrid LBA/LCMS assay and LBA assay can be used interchangeably, it is recommended that correlation and Bland-Altman plots be used. When changing to a uniplex Hybrid LBA/LCMS assay from a validated multiplexed LBA assay, some cross-validation tests are recommended to establish assay data comparability.

3. Overall, Hybrid LBA/LCMS ADA assays can be an alternative for screening, isotyping, and titration assays without needing a confirmatory assay. In cases where there is interference from drug or from endogenous substances, there could be some benefits of using Hybrid LBA/LCMS ADA assays for mitigating drug tolerance issues and decreasing the false positive rate. The requirements for the validation of Hybrid LBA/LCMS ADA assays should be leveraged from the best practices and lessons learned from LBA ADA assays, since currently there are no regulatory guidelines specific for Hybrid LBA/LCMS approaches. Using a species-specific ADA positive control for validations can help establish detection limits during method validation, and is essential for Hybrid LBA/LCMS assays for surrogate peptide selection.

4. Hybrid LBA/LCMS can be successfully applied to semi-quantitative measurement of ADAs through direct measurement of IgGs, IgMs, IgAs, IgEs and IgDs and their respective subclasses bound to the drug, or indirect measurement of the drug bound to ADAs. However, more industry experience is still needed before this technology is more widely adopted. The way of determining ADA positives and negatives and interpretation of ADA data also needs to be reconsidered and reevaluated due to the different techniques in measuring ADA.

\section{PK assays}

1. Hybrid LBA/LCMS assays provide structural characterization information during $\mathrm{PK}$ analysis and improve the understanding of the analyte and biology involved in addition to quantification. Method development is generally faster compared with traditional LBA, and comes with improved selectivity and less stringent reagent requirements. It is also generally less prone to matrix interference due to high specificity offered by the detection step. Scientists ideally should consider developing LBA and Hybrid methods in parallel with a clear stopping point to deciding which one to proceed with based on available results. .

2. The high selectivity of LCMS assays can provide greater discrimination between interferences, modifications, substitutions, and unique signature peptides than that afforded by traditional LBA approaches. When traditional LBA strategies to avoid ADA interferences in PK assays do not solve the issues, it is recommended to use LCMS or Hybrid LBA/LCMS assays with harsher conditions (e.g., alkaline, acid or guanidine combo treatments) for ADA dissociations than those typically used for LBA. A clear understanding of the analytical interferences and limitations is important.

3. Beads have been the most commonly used solid state method of choice for Hybrid LBA/LCMS assays. On-bead digestion is recommended because of its ease and lack of extra step when compared to off-bead digestion. The best approach to test clinical Hybrid LBA/LCMS assays for ADA measurement, especially where humans have not yet been dosed, is to use pre-existing ADAs, animal ADAs as positive controls. Selectivity should be tested in both pooled control and pre-dose patient matrices; the effects of specific ligands, such as soluble targets, at expected physiological concentrations upon drug quantitation in spiked QCs should be examined; as well as the impact of added ADAs of known high titers. Recovery and digestion efficiency should be examined during method validation. Recovery results, when patient pre-dose samples are tested naive and spiked at the LLOQ concentrations, provide information on selectivity 
and matrix effects. Parallelism experiments should be performed during validation and potentially also with (pooled) incurred samples. The issue of reagent management for Hybrid LBA/LCMS assays requires considerations similar to LBA reagents including reagent stability, suitability, and lot-to-lot comparability.

4. Hybrid LBA/LCMS methods are increasingly used to identify, characterize and quantify biotherapeutic biotransformations using an integrated approach. Understanding biotransformations will help scientists determine the impact on efficacy, safety, PD, and PK, as well as help to correlate with in vitro monitoring. If biotransformations of biotherapeutics are present in quantities of more than $10 \%$ of the starting material, it is recommended to follow the MIST guidance regarding quantification recommendations. For quantities below $10 \%$, the minor modified biotherapeutic is likely to have less impact on the overall activity, depending on how important the biotransformation is to the biotherapeutic.

${ }^{1}$ Genentech, South San Francisco, CA, USA

${ }^{2}$ Merck \& Co., Inc., Rahway, NJ, USA

${ }^{3}$ Angelini Pharma, Pomezia, RM, Italy

${ }^{4}$ Boehringer-Ingelheim, Ridgefield, CT, USA

${ }^{5}$ GlaxoSmithKline, King of Prussia, PA, USA

${ }^{6}$ Pfizer, Andover, MA, USA

${ }^{7}$ Caprion Biosciences, Montreal, QC, Canada

${ }^{8}$ Austria AGES, Wien, Austria

${ }^{9}$ Health Canada, Ottawa, ON, Canada

${ }^{10} \mathrm{WHO}$, Geneva, Switzerland

${ }^{11}$ Italy AIFA, Rome, Italy

${ }^{12}$ US FDA, Silver Spring, MD, USA

${ }^{13}$ Bioanalytical Laboratory Services a Division of LifeLabs LP, Toronto, ON, Canada

${ }^{14}$ Japan MHLW-NIHS, Tokyo, Japan

\section{References}

1 Savoie N, Booth BP, Bradley T et al. 2008 White Paper: the 2nd Calibration and Validation Group Workshop on recent issues in good laboratory practice bioanalysis. Bioanalysis 1(1), 19-30 (2009).

2 Savoie N, Garofolo F, van Amsterdam P et al. 2009 White Paper on recent issues in regulated bioanalysis from the 3rd Calibration and Validation Group Workshop. Bioanalysis 2(1), 53-68 (2010).

3 Savoie N, Garofolo F, van Amsterdam P et al. 2010 White Paper on recent issues in regulated bioanalysis and global harmonization of bioanalytical guidance. Bioanalysis 2(12), 1945-1960 (2010).

4 Garofolo F, Rocci M, Dumont I et al. 2011 White Paper on recent issues in bioanalysis and regulatory findings from

\section{Acknowledgements}

The authors would like to acknowledge the US FDA, Europe EMA, UK MHRA, Austria AGES, Italy AIFA, Brazil ANVISA, Health Canada, Japan MHWL and WHO for supporting this workshop. S Richards (Sanofi), L Amaravadi (Sanofi/Genzyme), R Pillutla (Bristol-Myers Squibb), H Birnboeck (F. Hoffmann-La Roche Ltd.), F Garofolo (Angelini Pharma) for chairing the workshop and/or the white paper discussions. All the workshop attendees and members of the bioanalytical community who have sent comments and suggestions to complete this White Paper. W Garofolo, L Lu, X Wang, M Losauro, N Savoie, A Hernandez, K Kalaydjian and J. Conception for the assistance in the organization of the event. Future Science Group as a trusted partner.

\section{Financial \& competing interests disclosure}

The authors have no relevant affiliations or financial involvement with any organization or entity with a financial interest in or financial conflict with the subject matter or materials discussed in the manuscript. This includes employment, consultancies, honoraria, stock ownership or options, expert testimony, grants or patents received or pending, or royalties.

No writing assistance was utilized in the production of this manuscript.

${ }^{15}$ Celerion, Lincoln, NE, USA

${ }^{16} \mathrm{Q} 2$ Solutions, Ithaca, NY, USA

${ }^{17}$ Novartis, Basel, Switzerland

${ }^{18}$ Brazil ANVISA, Brasilia, Brazil

${ }^{19}$ Bristol-Myers Squibb, Princeton, NJ, USA

${ }^{20}$ UCB Biopharma, Braine L'Alleud, Belgium

${ }^{21}$ CFABS, Montreal, QC, Canada

${ }^{22}$ Formerly MedImmune, Gaithersburg, MD, USA; currently Incyte Corporation, Wilmington, DE, USA

${ }^{23}$ Covance, Indianapolis, IN, USA

${ }^{24}$ UK MHRA, London, UK

${ }^{25}$ Dutch MEB, Utrecht, The Netherlands

${ }^{26}$ Roche Pharma Research and Early Development, Roche Innovation Center, Basel, Switzerland

audits and inspections. Bioanalysis 3(18), 2081-2096 (2011).

5 DeSilva B, Garofolo F, Rocci M et al. 2012 White Paper on recent issues in bioanalysis and alignment of multiple guidelines. Bioanalysis 4(18), 2213-2226 (2012).

6 Stevenson L, Rocci M, Garofolo F et al. 2013 White Paper on recent issues in bioanalysis: "hybrid" - the best of LBA \& LC/MS. Bioanalysis 5(23), 2903-2918 (2013).

7 Fluhler E, Hayes R, Garofolo F et al. 2014 White Paper on recent issues in bioanalysis: a full immersion in bioanalysis (Part 1 - small molecules by LCMS). Bioanalysis 6(22), 3039-3049 (2014).

8 Dufield D, Neubert H, Garofolo F et al. 2014 White Paper on recent issues in bioanalysis: a full immersion in bioanalysis (Part 2 - hybrid LBA/LCMS, ELN \& regulatory agencies' input). Bioanalysis 6(23), 3237-3249 (2014). 
9 Stevenson L, Amaravadi L, Myler H et al. 2014 White Paper on recent issues in bioanalysis: a full immersion in bioanalysis (Part 3 - LBA and immunogenicity). Bioanalysis 6(24), 3355-3368 (2014)

10 Welink J, Fluhler E, Hughes N et al. 2015 White Paper on recent issues in bioanalysis: focus on new technologies and biomarkers (Part 1 - small molecules by LCMS). Bioanalysis 7(22), 2913-2925 (2015).

11 Ackermann B, Neubert H, Hughes N et al. 2015 White Paper on recent issues in bioanalysis: focus on new technologies and biomarkers (Part 2 - hybrid LBA/LCMS and input from regulatory agencies). Bioanalysis 7(23), 3019-3034 (2015).

12 Amaravadi L, Song A, Myler H et al. 2015 White Paper on recent issues in bioanalysis: focus on new technologies and biomarkers (Part 3 - LBA, biomarkers and immunogenicity). Bioanalysis 7(24), 3107-3124 (2015).

13 Bak MJ, Albrechtsen NW, Pedersen J, Holst JJ. Specificity and sensitivity of commercially available assays for glucagon-like peptide-1 (GLP-1): implications for GLP-1 measurements in clinical studies. Diabetes Obes. Metab. 16(11), 1155-1164 (2014).

14 Bak MJ, Albrechtsen NW, Pedersen J et al. Specificity and sensitivity of commercially available assays for glucagon and oxyntomodulin measurement in humans. Eur. J. Endocrinol. 170 (4), 529-538 (2014).

15 Lee AY, Chappell DL, Bak MJ et al. Multiplexed quantification of proglucagon-derived peptides by immunoaffinity enrichment and tandem mass spectrometry after a meal tolerance test. Clin. Chem. 62(1), 227-235 (2016).

16 Chen LZ, Roos D, Philip E. Development of an immunocapture-LC/MS assay for simultaneous ADA isotyping and semi-quantitation. J. Immunol. Res. 2016, (2016).

17 US Department of Health and Human Services, US FDA, Center for Drug Evaluation and Research, Biologics Evaluation and Research. Draft Guidance for Industry, Assay Development for Immunogenicity Testing of Therapeutic Proteins. Rockville, MD, USA (2009).

18 US Department of Health and Human Services, US FDA, Center for Drug Evaluation and Research, Biologics Evaluation and Research. Draft Guidance for Industry, Assay Development and Validation for Immunogenicity Testing of Therapeutic Proteins. Rockville, MD, USA (2016).

19 Onami I, Ayabe M, Murao N, Ishigai M. A versatile method for protein-based antigen bioanalysis in non-clinical pharmacokinetics studies of a human monoclonal antibody drug by an immunoaffinity liquid chromatography-tandem mass spectrometry. J. Chromatogr. A. 1334, 64-71 (2014).

20 Wang SJ, Wu ST, Gokemeijer J et al. Attribution of the discrepancy between ELISA and LC-MS/MS assay results of a PEGylated scaffold protein in post-dose monkey plasma samples due to the presence of anti-drug antibodies. Anal. Bioanal. Chem. 402(3), 1229-1239 (2012).

21 Chirmule N, Jawa V, Meibohm B. Immunogenicity to therapeutic proteins: impact on PK/PD and efficacy. AAPS J. 14(2), 296-302 (2012).
22 Sailstad JM, Amaravadi L, Clements-Egan A et al. A White Paper - consensus and recommendations of a global harmonization team on assessing the impact of immunogenicity on pharmacokinetic measurements. AAPS J. 16(3), 488-98 (2014).

23 Xu K, Liu L, Saad OM et al. Characterization of intact antibody-drug conjugates from plasma/serum in vivo by affinity capture capillary liquid chromatography-mass spectrometry. Anal. Biochem. 412(1), 56-66 (2011).

24 Kaur S, Xu K, Saad O. Analysis of antibody drug conjugates by bead-based affinity capture and mass spectrometry: US8541178 (2013).

25 US FDA. Guidance for Industry: Safety Testing of Drug Metabolites (2008).

26 ICH S3A Guideline: Note for Guidance on Toxicokinetics: the Assessment of Systemic Exposure in Toxicity Studies Questions and Answers (2016).

27 US Department of Health and Human Services, US FDA, Center for Drug Evaluation and Research, Center for Veterinary Medicine. Guidance for Industry, Bioanalytical Method Validation. Rockville, MD, USA (2001).

28 European Medicines Agency. Committee for Medicinal Products for Human Use (CHMP). Guideline on Bioanalytical Method Validation. EMEA/CHMP/ EWP/192217/2009. London, UK (2011).

29 Rocci M, Lowes S, Shoup R et al. 7th GCC Insights: incurred samples use; fit-for-purpose validation, solution stability, electronic laboratory notebook and hyperlipidemic matrix testing. Bioanalysis 6(20), 2713-2720 (2014).

30 US Department of Health and Human Services, US FDA, Center for Drug Evaluation and Research, Center for Veterinary Medicine. Draft Guidance for Industry, Bioanalytical Method Validation. Rockville, MD, USA (2013).

31 US Department of Health and Human Services, US FDA, Center for Drug Evaluation and Research. Draft Guidance for Industry, Bioequivalence Studies with Pharmacokinetic Endpoints for Drugs Submitted Under an ANDA. Rockville, MD, USA (2013).

32 US Department of Health and Human Services, US FDA, Center for Drug Evaluation and Research. Product-specific recommendations for generic drug development. http://www.fda.gov/Drugs/ GuidanceComplianceRegulatoryInformation/Guidances/ ucm075207.htm

33 Nicholson R, Lowes S, Caturla MC et al. Conference report: 6th GCC focus on LBA: critical reagents, positive controls and reference standards; specificity for endogenous compounds; biomarkers; biosimilars. Bioanalysis 4(19), 2335-2342 (2012).

34 Hayes R, LeLacheur R, Dumont I et al. 9th GCC closed forum: CAPA in regulated bioanalysis; method robustness, biosimilars, preclinical method validation, endogenous biomarkers, whole blood stability, regulatory audit experiences and electronic laboratory notebooks. Bioanalysis 8(6), 487-495 (2016).

35 General Chapter <1106> Immunogenicity Assays - Design and Validation of Immunoassays to Detect Anti-Drug Antibodies, 
US Pharmacopeia and the National Formulary (USP-NF) (2014).

36 Shankar G, Devanarayan V, Amaravadi L et al. Recommendations for the validation of immunoassays used for detection of host antibodies against biotechnology products. J. Pharm. Biomed. Anal. 48, 1267-1281 (2008).

37 Title 21 - Food and Drugs, Chapter I - Food and Drug Administration Department of Health and Human Services Subchapter A - General: Part 58 - Good Laboratory Practice for Nonclinical Laboratory Studies.

38 OECD Series on Principles of Good Laboratory Practice and Compliance Monitoring, Number 1: Principles on Good Laboratory Practice (1997).

39 ICH Guideline for Good Clinical Practice E6(R1) (1996).

40 European Medicines Agency. GCP Inspectors Working Group. Draft Reflection Paper on Guidance for Laboratories that Perform the Analysis or Evaluation of Clinical Trial Samples. EMA/INS/GCP/532137/2010 (2010).

41 World Health Organization. Good Clinical Laboratory Practice (GCLP) (2009).

42 World Health Organization. WHO Expert Committee on Specifications for Pharmaceutical Preparations. Fifties Report Geneva, Annex 9 Guidance for Organizations Performing In Vivo Bioequivalence Studies (2016). http://www.who.int/ medicines/publications/pharmprep/WHO_TRS_996_ annex09.pdf

43 World Health Organization. WHO Expert Committee on Specifications for Pharmaceutical Preparations. Fifties Report Geneva, Annex 5 WHO Guidance on Good Data and Record Management Practices (2016). http://www.who.int/ medicines/publications/pharmprep/WHO_TRS_996_ annex05.pdf

44 ANVISA. Technical Note No 07/2015 - Bioequivalence Studies for Vitamins, Brazilian Health Surveillance Agency, Brazil (2015). http://portal.anvisa.gov.br/ documents/506392/0/CETER000.pdf/ff22cf14-c67a-4d37$99 \mathrm{~d} 0-88 \mathrm{a} 74700 \mathrm{e} 2 \mathrm{a} 6$
45 ANVISA. Technical Note No 09/2015-Pharmacokinetic Interaction for Fixed Dose Combinations and Clinical Trial Approval, Brazilian Health Surveillance Agency, Brazil (2015). http://portal.anvisa.gov.br/documents/506392/0/ NOTA $\pm 009 \pm 2015$. pdf $/ 7$ ef000d6-a39a-4b06-bbab$4 \mathrm{c} 5 \mathrm{ed} 21 \mathrm{f} 4 \mathrm{db} 4$

46 ANVISA. Technical Note No 02/2015 - PK Studies for Biological Products, Brazilian Health Surveillance Agency, Brazil (2015). http://portal.anvisa.gov.br/ documents/33836/2593824/Nota \pm de \pm esclarecimento \pm $\mathrm{n} \% \mathrm{C} 2 \% \mathrm{BA} \pm 02 \pm \mathrm{de} \pm 2015 / \mathrm{c} 9423232$-fdf0-4e68-8435a7c663fda26a

47 ANVISA. Resolution RDC no. 27, May 17, 2012. Validation of Bioanalytical Methods, Brazilian Health Surveillance Agency, Brazil (2012). http://portal.anvisa.gov.br/legislacao\#/ visualizar/28854

48 Health Canada, Resource Management and Operations Directorate. Draft Guidance Document - Disclosure of Confidential Business Information Under Paragraph 21.1(3)(c) of the Food and Drugs Act. Ottawa, Ontario, Canada (2016).

49 Health Canada. Notice: Proposed Policy on Bioequivalence Standards for Highly Variable Drug Products. (TBD).

50 Health Canada, Bureau of Policy, Science and International Programs Enquiries. Post-notice of Compliance (NOC) Changes: Quality Document. Ottawa, Ontario, Canada (2016).

51 Health Canada, Bureau of Pharmaceutical Sciences Therapeutic Products Directorate. Notice: Clarification of Bioanalytical Method Validation Procedures (2015).

52 Health Canada, Bureau of Pharmaceutical Sciences Therapeutic Products Directorate. Addendum to Notice: Clarification of Bioanalytical Method Validation Procedures (2016). 